\title{
Nucleation and growth of nickel nanoclusters on graphene Moiré on Rh(111)
}

\author{
M. Sicot, ${ }^{1, a)}$ S. Bouvron, ${ }^{1}$ O. Zander, ${ }^{1}$ U. Rüdiger, ${ }^{1}$ Yu. S. Dedkov, ${ }^{2}$ and M. Fonin ${ }^{1}$ \\ ${ }^{1}$ Fachbereich Physik, Universität Konstanz, 78457 Konstanz, Germany \\ ${ }^{2}$ Fritz-Haber-Institut der Max-Planck Gesellschaft, 14195 Berlin, Germany
}

(Received 7 December 2009; accepted 9 February 2010; published online 4 March 2010)

\begin{abstract}
Regularly sized Ni nanoclusters (NCs) have been grown on a graphene Moiré on $\mathrm{Rh}(111)$. Using scanning tunneling microscopy, we determine that initial growth of $\mathrm{Ni}$ at $150 \mathrm{~K}$ leads to preferential nucleation of monodispersed NCs at specific sites of the Moiré superstructure. However, a defined long-range ordering of $\mathrm{NCs}$ with increasing coverage is not observed. Room temperature Ni deposition leads to the formation of flat triangular-shaped islands which are well-matched to the Moiré registry.
\end{abstract}

The development of advanced routes to fabricate highly ordered monodispersed metallic nanostructures is one of the key challenges in modern nanotechnology. Transition metal nanoclusters (NCs) have become of special scientific interest and technological importance since they exhibit unique electronic structure, size-dependent catalytic activity, and selectivity as well as magnetic properties that are distinctly different from the bulk. ${ }^{1}$ One of the promising ways to produce arrays of homogeneously distributed monodispersed NCs is to use a bottom-up approach where self-organization growth phenomena on template substrates are used. So far, regular arrays of NCs were assembled using surfaces such as alumina double layers on $\mathrm{Ni}_{3} \mathrm{Al},{ }^{2,3}$ vicinal $\mathrm{Au}(111)$ surfaces, ${ }^{4,5}$ reconstructed surfaces ${ }^{6,7}$ or $h$-BN nanomesh. ${ }^{8,9}$ Recently, graphene Moiré on close-packed metal surfaces like $\operatorname{Pt}(111),{ }^{10} \mathrm{Rh}(111),{ }^{11} \mathrm{Ru}(0001),{ }^{12,13}$ and $\operatorname{Ir}(111)^{14,15}$ has been suggested to be a good candidate for the templated growth of clusters arrays. Recent works demonstrate that superlattices of metallic clusters of Re, W, Pt, and Ir on such graphene Moiré can be realized effectively. ${ }^{15-18}$

In this letter, we report on the growth of nanometer-sized Ni clusters on graphene Moiré on Rh(111). Growth and organization of Ni NCs deposited at different temperatures are investigated by means of scanning tunneling microscopy (STM) at room temperature (RT). Although Ni NCs deposited at $150 \mathrm{~K}$ and at low coverage are homogeneously sized and adsorbed on well-defined sites, no regular arrays exhibiting a long-range order could be obtained upon increasing the surface coverage. For RT deposition, the formation of flat triangular-shaped Ni islands is observed.

The experiments were carried out in an ultra-high vacuum system (base pressure $1 \times 10^{-10} \mathrm{mbar}$ ) equipped with an Omicron variable temperature scanning probe microscope (VT SPM). Prior to graphene preparation, the $\mathrm{Rh}(111)$ single crystal was cleaned by repeated cycles of $\mathrm{Ar}^{+}$sputtering, annealing in oxygen, and subsequent flash annealing to $1400 \mathrm{~K}$. The cleanliness of the $\mathrm{Rh}(111)$ surface was verified via the observation of the surface state by ultraviolet photoemission spectroscopy (not shown here). An ordered graphene monolayer (ML) on $\mathrm{Rh}(111)$ was prepared via cracking of propene $\mathrm{C}_{3} \mathrm{H}_{6}$ gas as described elsewhere. ${ }^{11} \mathrm{Ni}$ was evaporated from a high-purity piece heated by electron

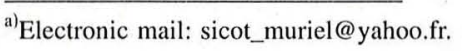

bombardment at a deposition rate of about $1 \AA / \mathrm{min}$ on samples held at $150 \mathrm{~K}$ or RT. Surface coverage is given in ML where $1 \mathrm{ML}$ corresponds to the surface atomic density of $\mathrm{Rh}(111)$. Electrochemically etched tungsten (W) tips were flash annealed by electron bombardment in situ and used for STM imaging. The sign of the bias voltage corresponds to the voltage applied to the sample.

The quality of the graphene surface over macroscopic distances was checked by low energy electron diffraction (LEED). A typical LEED pattern of the graphene Moire on $\mathrm{Rh}(111)$ is given in Fig. 1(a). From LEED images a periodicity of $2.90 \pm 0.05 \mathrm{~nm}$ of the Moiré superstructure on $\mathrm{Rh}(111)$ was calculated. The high-resolution STM topograph of the Moiré pattern in Fig. 1(b) clearly reveals a defect free single domain. The distance between the neighboring white protrusions within the hexagonal lattice was measured to be about $3 \mathrm{~nm}$ supporting the results of the LEED measurements. The lattice mismatch between graphene and the $\mathrm{Rh}(111)$ surface causes a gentle corrugation of the graphene sheet as indicated by the line profile in Fig. 1(c). Figure 1(d) displays an atomically resolved STM topograph of the
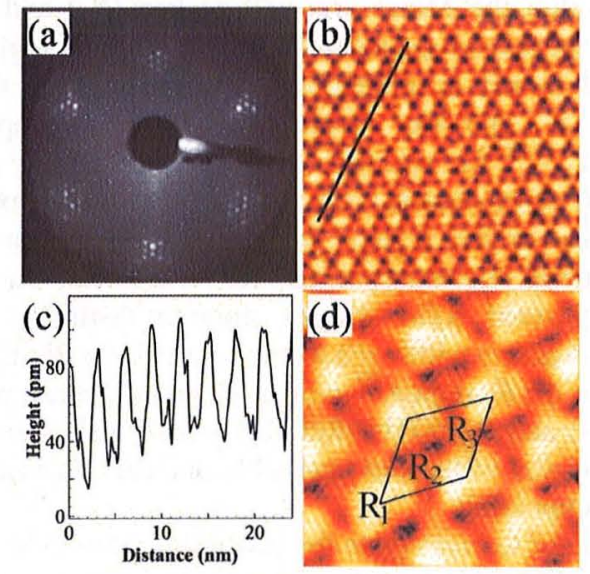

FIG. 1. (Color online) (a) A LEED pattern of the graphene/Rh(111) surface taken at an electron beam energy of $87.5 \mathrm{eV}$. (b) $35 \times 35 \mathrm{~nm}^{2}$ STM topograph of graphene Moiré on $\mathrm{Rh}(111)$. (c) A height profile along the line indicated in (b). (d) $10 \times 10 \mathrm{~nm}^{2}$ atomically resolved STM topograph of graphene on $\mathrm{Rh}(111)$. The rhombus outlines the supercell of the Moire superstructure with different regions marked by $R_{1}, R_{2}$, and $R_{3}$. Tunneling parameters: (b) $+1 \mathrm{~V}, 0.5 \mathrm{nA}$ and (d) $+10 \mathrm{mV}, 2.5 \mathrm{nA}$. 

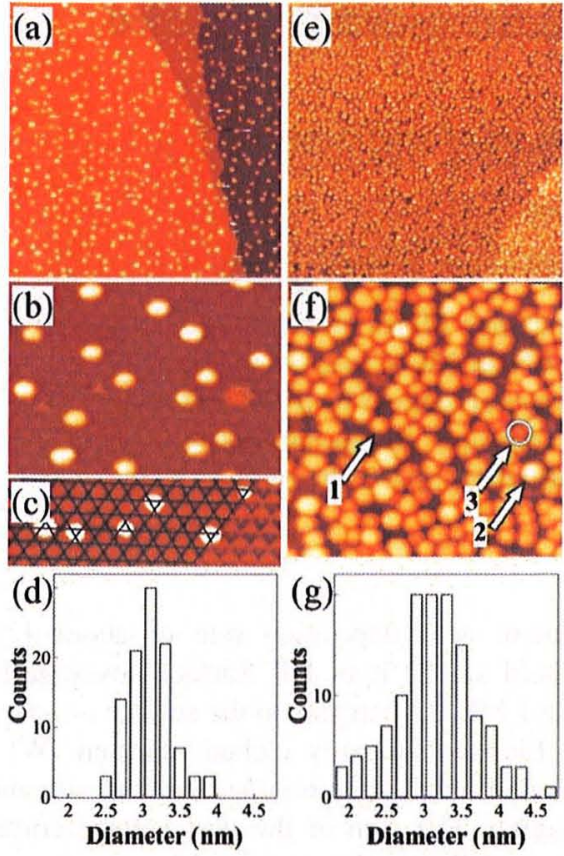

FIG. 2. (Color online) STM topographs obtained after deposition of 0.25 $\mathrm{ML} \mathrm{Ni}$ at $150 \mathrm{~K}$ (a), (b), (c), and $1.50 \mathrm{ML} \mathrm{Ni}$ (e), (f). The corresponding histograms of clusters diameter vs the number of clusters are displayed in [(d) and $(\mathrm{g})]$. The Moiré superstructure in (c) is outlined by a grid. Numbers in (f) refer to different kinds of NCs. Image sizes are (a) and (e) 200 $\times 200 \mathrm{~nm}^{2}$; (b) $50 \times 35 \mathrm{~nm}^{2}$; (c) $50 \times 15 \mathrm{~nm}^{2}$; and (f) $50 \times 50 \mathrm{~nm}^{2}$. Tunneling parameters: (a) $+2 \mathrm{~V}, 0.14 \mathrm{nA}$; (b) $+1.02 \mathrm{~V}, 0.13 \mathrm{nA}$; (c) $+0.95 \mathrm{~V}$, $1.56 \mathrm{nA}$; (e) $+1.12 \mathrm{~V}, 2 \mathrm{nA}$; and (f) $+1.29 \mathrm{~V}, 0.8 \mathrm{nA}$

graphene surface. The rhombic unit cell of the Moiré pattern is outlined in the STM image [Fig. 1(d)]. On other metallic close-packed surfaces ${ }^{10,13,14}$ large bright protrusions and darker regions in between are interpreted as areas where carbon atoms are positioned at atop sites and $f c c / h c p$ sites, respectively. Following this notation, we identify the pronounced light areas $\left(R_{1}\right)$ as atop regions and two distinct areas within the Moiré unit cell as $h c p\left(R_{2}\right)$ and $f c c\left(R_{3}\right)$ regions. In case of graphene on $\operatorname{Ir}(111),{ }^{16,19}$ hcp regions were reported to act as preferential adsorption sites for metal (Ir, Pt, W, and Re) clusters whereas for graphene on $\mathrm{Ru}(0001),{ }^{17,18} f c c$ regions were identified as the preferred locations of Pt clusters. One can also reasonably expect that either $h c p$ or $f c c$ regions of graphene Moiré on $\mathrm{Rh}(111)$ would act as nucleation centers for Ni clusters. On the other hand, obtained STM images show a distinctly different atomic structure within the Moiré unit cell on $\mathrm{Rh}(111)$, especially for the $R_{2}$ region, ${ }^{20}$ compared with graphene on $\operatorname{Ir}(111)$ (Ref. 14) and $\mathrm{Ru}(0001)$ (Ref. 12) which makes an a priori determination of the most probable nucleation centers for $\mathrm{Ni}$ cluster impossible.

In order to investigate the possibility to use the graphene Moiré on $\mathrm{Rh}(111)$ as a template for the growth of ordered arrays of magnetic NCs, we deposited Ni at $150 \mathrm{~K}$. Growth at low temperature was chosen in order to suppress the high mobility of small clusters and thus to induce an ordered array formation. Figure 2(a) shows an STM topograph of 0.25 ML of $\mathrm{Ni}$ deposited on graphene/Rh(111) at $150 \mathrm{~K}$. Large terraces and steps of graphene/Rh(111) covered with $\mathrm{Ni} N \mathrm{NCs}$ can be clearly distinguished. Figure 2 (b) shows a magnified STM topograph of a terrace area with Ni NCs on top of the
Moiré superstructure. The NCs show mostly hemispherical shapes with at least some cluster edges which appear to be oriented along the in-plane $\langle 1 \overline{1} 0\rangle$ direction. The apparent height of the clusters was measured to be $0.85 \pm 0.1 \mathrm{~nm}$ which roughly corresponds to four $f c c$ (111) planes of $\mathrm{Ni}$. The clusters are loosely bound to the graphene surface at RT and could easily be swept away by the STM tip during scanning. The diameter distribution of the clusters is very narrow as shown by the histogram in Fig. 2(d) and the average cluster diameter (full width at half maximum) is $3.1 \mathrm{~nm}$. The narrow distribution together with the average cluster diameter of about $3 \mathrm{~nm}$ is a clear indication of the geometric confinement provided by the template. In order to address a possible registry with the Moiré, a grid corresponding to the Moiré superstructure is superimposed on a smaller scale topograph in Fig. 2(c). Most of the Ni clusters are located on the regular grid showing a quite high unit cell occupation probability for the deposited amount. However, the nucleation of NCs obviously occurs at two different sites, namely the $R_{2}$ and $R_{3}$ regions within the Moiré unit cell with the occupation ratio of about two to one. The randomized occupation can be attributed to the fact that the deposition temperature of $150 \mathrm{~K}$ is below the optimum temperature. This leads to an effective occupation of both regions within the Moiré unit cell upon suppressed surface mobility. However, deposition at higher temperatures does not lead to increased selective occupation and formation of ordered arrays but rather to the formation of large epitaxial islands. Moreover, in order to determine the sites of enhanced reactivity toward binding metal atoms one should take into account the bonding strength between the graphene layer and the $\mathrm{Rh}(111)$ surface. The stronger bonding of the graphene layer to the metallic substrate was shown to result in local $s p^{2}$ to $s p^{3}$ rehybridization of the $\mathrm{C}-\mathrm{C}$ bonds leading to the preferential adsorption of metal atoms on top of the graphene layer. ${ }^{19}$ In contrast to graphene on $\mathrm{Ru}(0001)$, in the present study we observe an additional bright feature centered in the $R_{2}$ region [Fig. 1(d)] corresponding to a weakly bound area which coincides well with the trend where the strength of covalent bonding decreases in the series Ru-Rh-Ir. The overall structural difference between the $f c c$ and $h c p$ regions is less pronounced which might lead to comparable reactivity for both regions. Detailed theoretical calculations are necessary to resolve these issues. We would like to note here, that the occupation statistics or even the growth mode can change depending on the deposited material. ${ }^{16}$ Moreover, kinetic factors such as deposition rate may also play an important role.

Upon increasing $\mathrm{Ni}$ coverage no visible order of the cluster arrangement can be realized. Figure 2(e) shows the surface morphology after the deposition of $1.50 \mathrm{ML}$ of $\mathrm{Ni}$ at 150 K. In Fig. 2(f), clusters exhibit a distinctly different size distribution compared with that at lower coverages. The lateral size of the clusters is more spread as shown by the corresponding diameter histogram in Fig. 2(g). One can distinguish three kinds of clusters which will be referred to as type 1,2 , and 3 in the following and which are pointed by arrows in Fig. 2(f). Clusters of type 1 exhibit shape and size similar to those observed at lower coverages, i.e., typically $3 \mathrm{~nm}$ in diameter and $4 \mathrm{ML}$ high. Clusters of type 2 are of hexagonal shape and extend over more than one Moiré unit cell. The majority of clusters of type 2 are 5 ML high and 


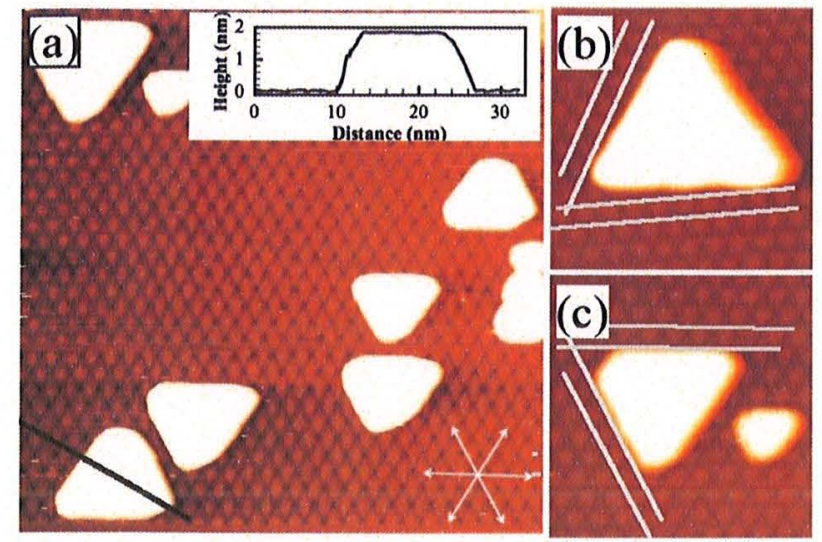

FIG. 3. (Color online) (a) $85 \times 85 \mathrm{~nm}^{2} \mathrm{STM}$ topograph of $0.90 \mathrm{ML} \mathrm{Ni}$ on graphene/Rh(111) deposited at RT. The arrows indicate the close-packed directions of the $\operatorname{Rh}(111)$ substrate. The inset displays the profile along the black line marked in the figure. (b) and (c) show $35 \times 35 \mathrm{~nm}^{2}$ STM toporgaphs of a $4^{\circ}$-rotated and unrotated $\mathrm{Ni}$ island, respectively. Tunneling parameters: (a), (c) $+0.9 \mathrm{~V}, 0.88 \mathrm{nA}$; and (b) $+1.45 \mathrm{~V}, 0.25 \mathrm{nA}$.

only a small fraction is $6 \mathrm{ML}$ high. Clusters of type 3 look squeezed between bigger neighboring clusters. They have a size of about $2.5 \mathrm{~nm}$ or less and a height of $3 \mathrm{ML}$. The absence of an ordered cluster array for $\mathrm{Ni}$ at higher coverage is somehow expectable due to the observed random occupation of two different sites within the Moiré unit cell which can be attributed to a small difference in the depth of the potential energy minima within a Moiré unit cell being not sufficient to selectively trap adatoms and small clusters on a unique site.

Ni deposited at RT exhibits a completely different growth mode compared with deposition at $150 \mathrm{~K}$. Instead of small compact $\mathrm{NCs}$, Ni forms triangular-shaped islands with their edges roughly aligned with the close packed $\langle 1 \overline{1} 0\rangle$ directions of the $\mathrm{Rh}$ (111) substrate as shown in Fig. 3(a). The existence of large islands nucleated at terraces indicates that $\mathrm{Ni}$ atoms are highly mobile on the graphene surface at RT. On the other hand, it is remarkable that, in spite of the weak bonding strength between $\mathrm{Ni}$ and graphene surface, the Moiré structure imposes registration and orientation on the Ni nanostructures. The average apparent height of the islands is $1.8 \mathrm{~nm}$ and the size defined by the length of the edges is ranging from about $5 \mathrm{~nm}$ to about $18 \mathrm{~nm}$. The surface of the islands is rather flat as shown by the line profile in Fig. 3(a). The two different orientations can be attributed to the initial nucleation taking place either at a $f c c$ or an $h c p$ site. In order to fully describe the growth mode, additional studies including variation of deposition temperature as well as the growth rate should be performed and compared with the existing models. ${ }^{21}$

Interestingly, the long edges of the islands are not always exactly aligned with the high-symmetry $\langle 1 \overline{1} 0\rangle$ directions of $\mathrm{Rh}(111)$. Some of the islands are oriented with an angle of up to $\pm 10^{\circ}$ with respect to these high-symmetry directions. Given the residual uncertainties in scanner calibration we estimate the uncertainty of the angles to be $\pm 1^{\circ}$. The examples of both island types rotated and unrotated are presented in Figs. 3(b) and 3(c), respectively. Clockwise-rotated and counterclockwise-rotated islands are found to occur with equal probability. As only one orientation can have the lowest energy, some kinetic mechanism is responsible for the existence of different orientations. For example, the nucleation event might lead to the selection of the island orientation with the orientational dependence of the energy being too small to allow subsequent equilibration.

In conclusion, Ni NCs have been grown on a graphene Moiré on $\mathrm{Rh}$ (111) and studied by STM at RT. At a deposition temperature of $150 \mathrm{~K}$ and low coverage, the size of Ni NCs is about $3 \mathrm{~nm}$ and is monodispersed suggesting the efficient geometrical confinement induced by the Moiré structure. Although we found a trend in the occupation of specific adsorption sites of the graphene Moiré at lower coverages, no organization of the clusters is observed when increasing coverage. We demonstrated that RT Ni deposition leads to the formation of flat triangular-shaped islands which are well-matched to the Moiré registry. Since the magnetic anisotropy depends on shape and size of the cluster, the deposition temperature is the key parameter to play with in order to control the magnetic properties of the nanoparticles.

This work was supported by the Research Center "UltraQuantum" (Excellence Initiative). Part of this work was supported by the Deutsche Forschungsgemeinschaft (DFG) via the Collaborative Research Center (SFB) 767.

${ }^{1}$ Metal Clusters at Surfaces, Structure, Quantum Properties, Physical Chemistry, edited by K.-H. Meiwes-Broer (Springer, Berlin, 2000).

${ }^{2}$ S. Degen, C. Becker, and K. Wandelt, Faraday Discuss. 125, 343 (2004).

${ }^{3}$ M. Schmid, G. Kresse, A. Buchsbaum, E. Napetschnig, S. Gritschneder, M. Reichling, and P. Varga, Phys. Rev. Lett. 99, 196104 (2007).

${ }^{4}$ V. Repain, G. Baudot, H. Ellmer, and S. Rousset, Europhys. Lett. 58, 730 (2002).

${ }^{5}$ N. Weiss, T. Cren, M. Epple, S. Rusponi, G. Baudot, S. Rohart, A. Tejeda, V. Repain, S. Rousset, P. Ohresser, F. Scheurer, P. Bencok, and H. Brune, Phys. Rev. Lett. 95, 157204 (2005).

${ }^{6}$ M. Fonin, Y. S. Dedkov, U. Rüdiger, and G. Güntherodt, Surf. Sci. 529, L275 (2003).

${ }^{7}$ W. Chen, K. P. Loh, H. Xu, and A. T. S. Wee, Appl. Phys. Lett. 84, 281 (2004).

${ }^{8}$ W. Auwärter, M. Muntwiler, T. Greber, and J. Osterwalder, Surf. Sci. 511, 379 (2002).

9J. Zhang, V. Sessi, C. H. Michaelis, I. Brihuega, J. Honolka, K. Kern, R. Skomski, X. Chen, G. Rojas, and A. Ender, Phys. Rev. B 78, 165430 (2008).

${ }^{10}$ T. Land, T. Michely, R. Behm, J. Hemminger, and G. Comsa, Surf. Sci. 264, 261 (1992).

${ }^{11}$ A. B. Preobrajenski, M. L. Ng, A. S. Vinogradov, and N. Mårtensson, Phys. Rev. B 78, 073401 (2008).

${ }^{12}$ S. Marchini, S. Günther, and J. Wintterlin, Phys. Rev. B 76, 075429 (2007).

${ }^{13}$ A. L. Vázquez de Parga, F. Calleja, B. Borca, J. M. C. G. Passeggi, J. J. Hinarejos, F. Guinea, and R. Miranda, Phys. Rev. Lett. 100, 056807 (2008).

${ }^{14}$ A. T. N'Diaye, J. Coraux, T. N. Plasa, C. Busse, and T. Michely, New J. Phys. 10, 043033 (2008).

${ }^{15}$ A. T. N'Diaye, T. Gerber, C. Busse, J. Mysliveček, J. Coraux, and T. Michely, New J. Phys. 11, 103045 (2009).

${ }^{16}$ A. T. N'Diaye, S. Bleikamp, P. J. Feibelman, and T. Michely, Phys. Rev. Lett. 97, 215501 (2006).

${ }^{17}$ Y. Pan, M. Gao, L. Huang, F. Liu, and H.-J. Gao, Appl. Phys. Lett. 95, 093106 (2009).

${ }^{18} \mathrm{~K}$. Donner and P. Jakob, J. Chem. Phys. 131, 164701 (2009).

${ }^{19}$ P. J. Feibelman, Phys. Rev. B 77, 165419 (2008).

${ }^{20}$ The details of the atomic arrangement are out of scope of the present work and will be reported separately.

${ }^{21}$ H. Brune, Surf. Sci. Rep. 31, 125 (1998). 